\title{
Two-Component Assembly of Thiochroman-4-ones and Tetrahydrothiopyran-4-ones Using a Rhodium-Catalyzed Alkyne Hydroacylation/Thio-Conjugate-Addition Sequence
}

\author{
Anaïs Bouisseau, John Glancy, and Michael C. Willis*
}

Department of Chemistry, University of Oxford, Chemistry Research Laboratory, Mansfield Road, Oxford, OX1 3TA, U.K.

Supporting Information
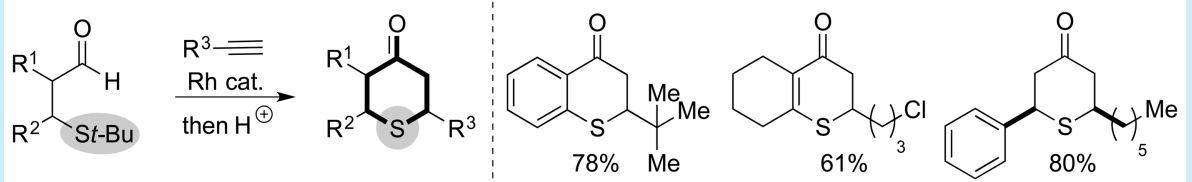

ABSTRACT: $\beta^{\prime}$-Thio-substituted-enones, assembled from the combination of $\beta$-tert-butylthio-substituted aldehydes and alkynes, using rhodium catalysis, are shown to smoothly undergo in situ intramolecular $S$-conjugate addition to deliver a range of $S$-heterocycles in a one-pot process. Aryl, alkenyl, and alkyl aldehydes can all be employed, to provide thiochroman-4-ones, hexahydro- $4 H$-thiochromen-4-ones, and tetrahydrothiopyran-4-ones, respectively. A variety of in situ oxidations are also performed, allowing access to $S, S$-dioxide derivatives, as well as unsaturated variants.

$\mathrm{R}$ ecent years have seen the emergence of alkene and alkyne hydroacylation reactions as useful methods for the synthesis of ketones and enones, respectively. ${ }^{1}$ For metalcatalyzed variants, ${ }^{2-5}$ strategies based on the use of some form of chelation control, with the coordinating group pendant to the aldehyde ${ }^{6-10}$ or alkene/alkyne, ${ }^{11}$ have been shown to deliver efficient, selective reactions, ${ }^{6 c, 7 b, 12,11 b, 13}$ with a generally broad substrate scope. The inherent limitation of a chelationcontrolled approach is that the coordinating substituent, which is crucial to achieve a successful reaction, is inevitably present in the product. Approaches to mitigate this situation include exploiting the coordinating group in subsequent catalystcontrolled transformations, ${ }^{14}$ and also incorporating the group, usually via derivatization, into a target structure. In the context of this latter strategy, our laboratory demonstrated that dihydroquinolones could be conveniently prepared from an alkyne hydroacylation/ $N$-conjugate addition sequence commencing with 2-aminobenzaldehyde substrates. ${ }^{7 c}$ More recently, the Stanley group reported the development of a tandem alkyne hydroacylation/oxa-Michael addition process to synthesize trans-2,3-disubstituted chroman-4-ones from $O$ chelating benzaldehydes (Scheme 1a). ${ }^{15}$ In this Letter we show that $S$-chelating aldehydes can be used to access a variety of $S$-heterocycles from related hydroacylation/conjugate addition sequences (Scheme 1b). A crucial difference with the present study is that the use of $S$-chelating aldehydes allows access to heterocycles of much greater variety than either the $\mathrm{N}$-or $\mathrm{O}$-based chemistries.

Thiochromanones and related heterocycles, notably $S, S$ dioxide derivatives and unsaturated variants, are present in a number of medicinally relevant molecules (Scheme 1c), ${ }^{16}$ and as such we envisaged a general synthetic route that would allow access to a broad range of related $S$-heterocycles. Our approach is shown in Scheme $1 \mathrm{~b}$ and involves chelate-controlled alkyne hydroacylation followed by an acid triggered thio-conjugate addition. ${ }^{17}$ Although encouraging precedent is provided from our own reports of hydroquinolone synthesis, ${ }^{7 \mathrm{c}}$ and Stanley's chroman-4-one chemistry, ${ }^{15}$ both of these sequences are limited to aryl aldehyde substrates, thus constraining the structural variation accessible in the products. Of the chelating aldehydes that have been reported, $S$-substituted examples allow arguably the most structural variation with alkyl, ${ }^{8 a}$ alkenyl, $^{12 \mathrm{c}}$ aryl, ${ }^{8 \mathrm{c}}$ and heteroaryl ${ }^{18}$ derivatives all being competent substrates. We reasoned that exploiting this feature of $S$-chelation control would allow access to not only thiochromanones but also the related saturated and partially saturated derivatives.

Our general approach requires aldehyde substrates bearing a sulfide substituent that would ideally be cleaved under the cyclization conditions. Given the proposed acid mediated cyclization (i.e., I $\rightarrow$ II $\rightarrow$ III, Scheme 1 b) we settled on the use of $t$-Bu-sulfides. ${ }^{19}$ Although $\beta$ - $t$-Bu- $S$-substituted aldehydes have never been previously reported as substrates for alkene or alkyne hydroacylation, we were pleased to find that the use of established reaction conditions, developed mainly for $\beta$-Me-Ssubstituted variants, led to efficient hydroacylation. ${ }^{20}$ For example, coupling between 2-tert-butyl-sulfide-substituted benzaldehyde 1a and 4-tolylacetylene using $5 \mathrm{~mol} \%$ of commercially available $\mathrm{Rh}(\mathrm{nbd})_{2} \mathrm{BF}_{4}$ and the dcpm ligand in 1,2-dichloroethane at $55{ }^{\circ} \mathrm{C}$ resulted in quantitative formation of the enone product. A short investigation of possible cyclization conditions established that in situ treatment of the

Received: September 27, 2016

Published: October 25, 2016 
Scheme 1. Tandem Hydroacylation/Conjugate Addition Sequences Towards Heterocycles, Together with Biologically Relevant $S$-Heterocycles

a) Tandem alkyne hydroacylation/oxa-Michael addition (Stanley, 2015)

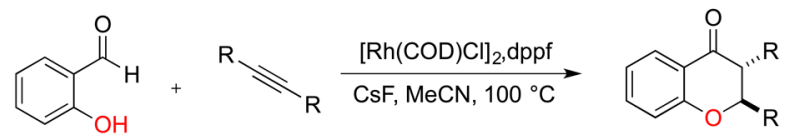

limited to phenolic

aldehydes as substrates

b) Tandem alkyne hydroacylation/thio-conjugate addition (this work)

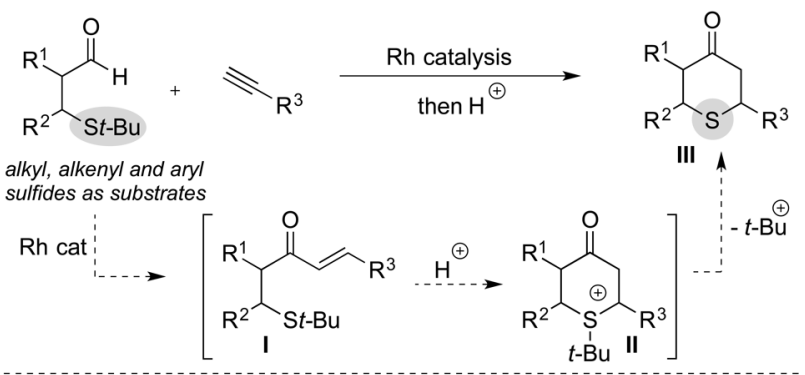

c) Examples of biologically active thiochroman-4-ones and derivatives

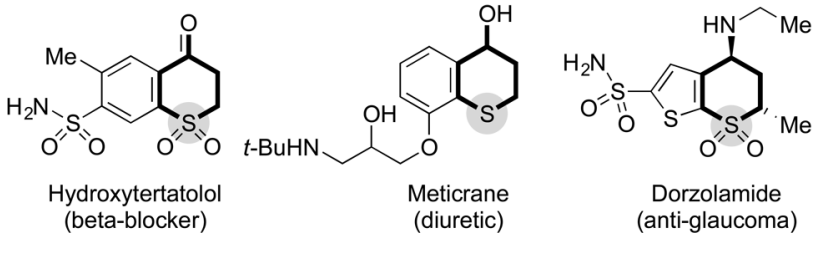

hydroacylation adduct with excess trifluoroacetic acid (TFA) resulted in clean conversion to the $S$-heterocycle.

Scheme 2 shows our exploration of these reaction conditions using aldehyde $1 \mathrm{a}$ in combination with a range of alkyne substrates (2). The transformation was successfully applied to phenyl acetylene derivatives bearing a variety of electrondonating $(3 \mathbf{a}-\mathbf{c})$ and electron-withdrawing groups $(3 \mathbf{d}-\mathbf{g})$. Substituents on the alkyne moiety were tolerated at all positions of the benzene rings. Notable among those examples is $3 \mathbf{c}$, which has been reported as a potent antioxidant displaying high inhibitory activity against nitric oxide production. ${ }^{16 f}$ The tandem reaction was also achieved employing reactive cyano groups $(\mathbf{3 g}, \mathbf{3 o})$ and a simple heterocycle (3h). Alkynes possessing aliphatic chains also afforded thiochroman-4-ones in excellent yields $(3 \mathbf{i}-\mathbf{o})$, even when employing a bulky tert-butyl substituent (31). Pleasingly, the tandem process was also found to be applicable on a gram scale reaction using 4-tolylacetylene, with only $1 \mathrm{~mol} \%$ of rhodium catalyst and ligand delivering the final product in comparable yield.

We next examined the scope of the aldehyde component (Scheme 3). A variety of 2-(tert-butylthio)benzaldehyde derivatives (1) were combined with 4-tolylacetylene, delivering diversely substituted thiochroman-4-ones possessing electrondonating (3p, 3q, 3u) and electron-withdrawing $(3 \mathbf{r}-\mathbf{u})$ functional groups in moderate to excellent yields. The use of an alkenyl derived $S$-chelating aldehyde led to thiochromen-4one $3 \mathbf{v}$ in good yield. Pleasingly, tetrahydrothiopyran-4-ones could also be accessed $(3 \mathbf{w}-\mathbf{y})$ with acceptable yields and diastereoselectivities. The tandem process was also found to be applicable to internal alkynes, enabling the formation of tetrahydrothiopyran-4-one $3 z$ featuring three stereogenic centers. Employing the same internal alkyne in combination with aromatic aldehyde 1a was also a successful reaction;
Scheme 2. Variation of Alkyne Structure in the Formation of Thio-4-chromanones ${ }^{a}$

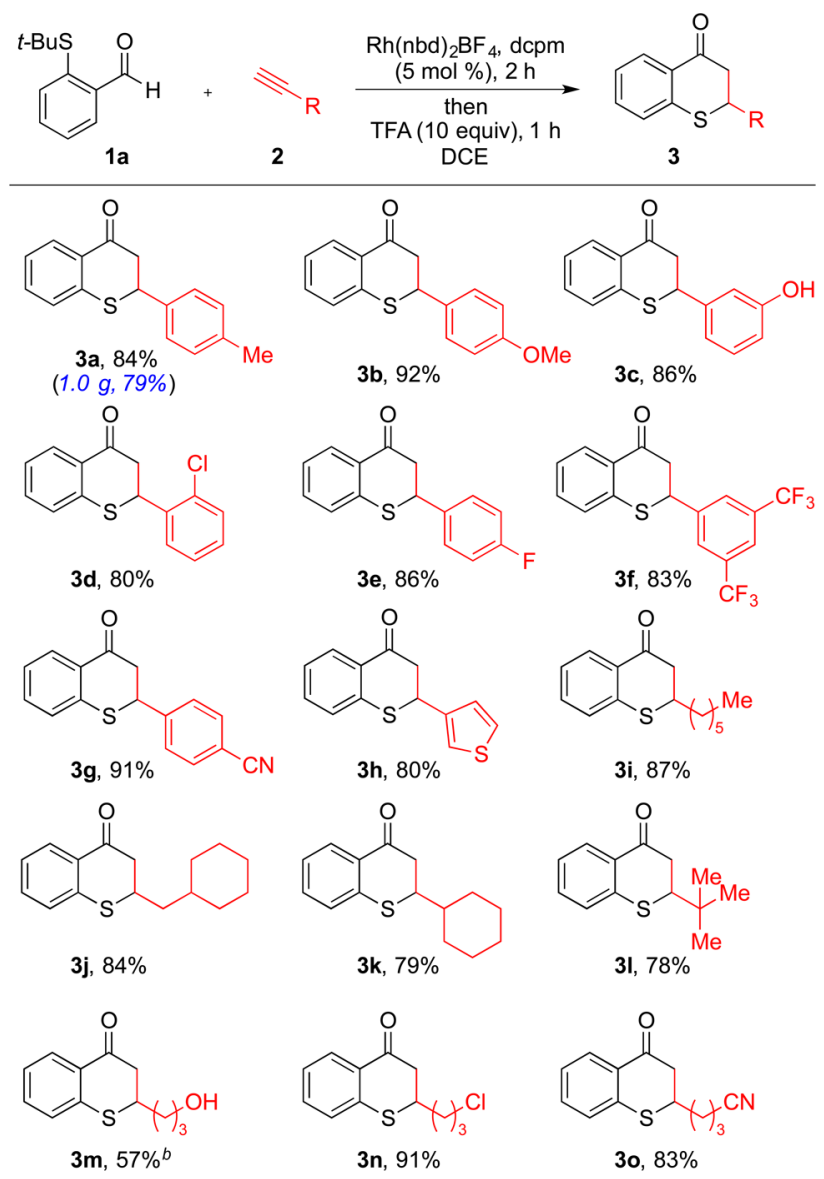

${ }^{a}$ Reaction conditions: $\mathrm{Rh}(\mathrm{nbd}){ }_{2} \mathrm{BF}_{4}$ (5 mol \%), dcpm (5 mol \%), aldehyde 1a (1.0 equiv), alkyne (1.05 equiv), DCE $(1.0 \mathrm{M}), 55^{\circ} \mathrm{C}, 2$ $\mathrm{h}$, then TFA (10.0 equiv), $1 \mathrm{~h}$, rt. ${ }^{b} \mathrm{~K}_{2} \mathrm{CO}_{3}, \mathrm{MeOH}, 16 \mathrm{~h}$, rt, after acid step. Isolated yields.

however, in this case the disubstituted thiochroman-4-one (3aa) was obtained as an inseparable 1:1.8 mixture of diastereomers. This level of diastereoselectivity is consistent with that seen by Stanley for the corresponding chroman-4-one series. $^{15}$

Aware that the $S, S$-dioxide derivatives of thiochromanones and tetrahydrothiopyranones are of interest as medicinal agents, we adapted our process to allow access to this class of compounds directly (Scheme 4). Simply adding an aqueous solution of hydrogen peroxide at the completion of the cyclization step resulted in oxidation to the desired sulfone derivatives. Aryl- and alkyl-substituted thiochromanones (4a,b), together with an alkyl-substituted tetrahydrothiopyranone (4c), provided the oxidized products in moderate to good yields.

We developed a further modification of our hydroacylationbased route to $S$-heterocycles, whereby addition of $N$ chlorosuccinimide (NCS) subsequent to $S$-conjugate addition allowed access to thiochromenone and dihydrothiopyran-4-one derivatives (Scheme 5). Following a report from Chen, ${ }^{21}$ we investigated the addition of NCS directly to the reaction mixtures. However, the excess TFA used to promote the $S$ cyclization was incompatible with this approach. The solution was to remove the excess TFA in vacuo before addition of the NCS and pyridine to the crude reaction mixture. Using this 
Scheme 3. Variation of Aldehyde Structure in the Formation of Thiochroman-4-ones, Thiochromen-4-ones, and Tetrahydrothiopyran-4-ones ${ }^{a}$

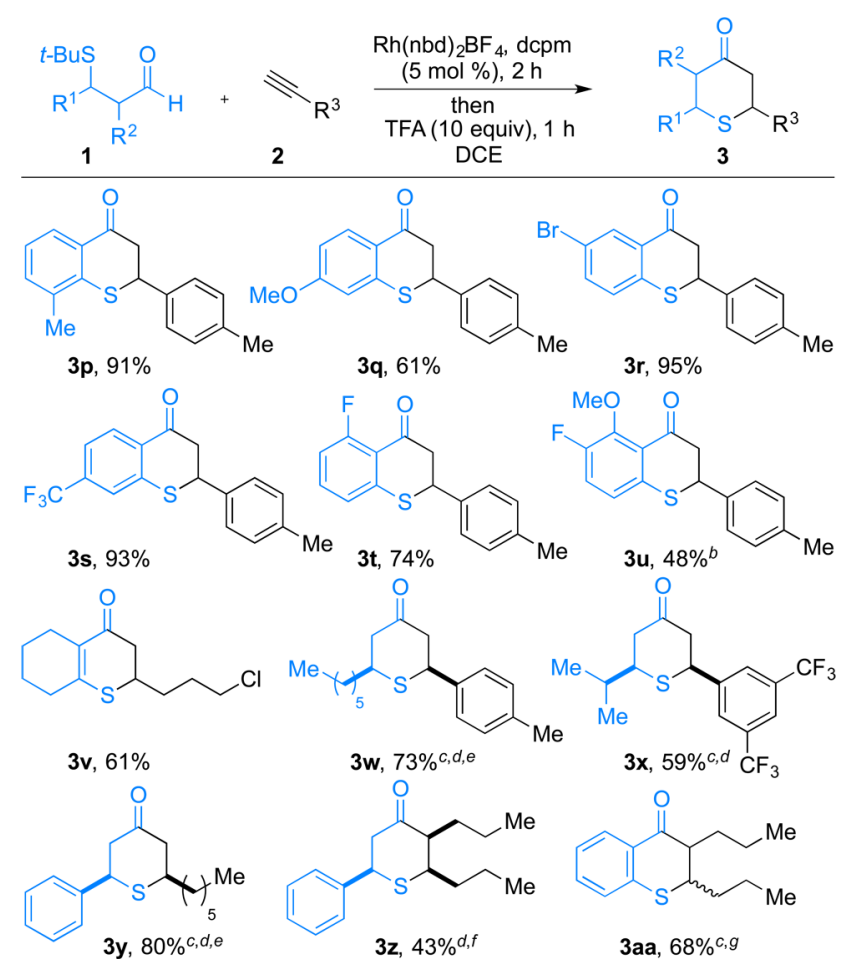

${ }^{a}$ Isolated yields of pure diasteromers. Reaction conditions: Rh$(\mathrm{nbd})_{2} \mathrm{BF}_{4}(5 \mathrm{~mol} \%), \mathrm{dcpm}(5 \mathrm{~mol} \%)$, aldehyde (1.0 equiv), alkyne (1.05 equiv), DCE $(1.0 \mathrm{M}), 55^{\circ} \mathrm{C}, 2 \mathrm{~h}$, then TFA (10.0 equiv), $1 \mathrm{~h}$, rt. ${ }^{b} \mathrm{Rh}(\mathrm{nbd})_{2} \mathrm{BF}_{4}$ and dcpm (both $7.5 \mathrm{~mol} \%$ ). ${ }^{c}$ alkyne (1.2 equiv). ${ }^{d}$ Crude dr values: $3 \mathrm{w}: 15: 1,3 \mathrm{x}: 19: 1,3 \mathrm{y}: 17: 1,3 \mathrm{z}: 11.5: 2.1: 1.5: 1.0$. ${ }^{e}$ Product contains traces of the minor diastereomer. ${ }^{f} \mathrm{Rh}(\mathrm{nbd})_{2} \mathrm{BF}_{4}$ and dppm (both $10.0 \mathrm{~mol} \%$ ), alkyne (1.5 equiv), DCE (1.0 M), $80{ }^{\circ} \mathrm{C}, 2$ h. ${ }^{g}$ dppm $(5 \mathrm{~mol} \%)$ used in place of dcpm. Isolated as an inseparable 1:1.8 mixture of diastereomers.

Scheme 4. In Situ Preparation of S,S-Dioxide Derivatives ${ }^{a}$

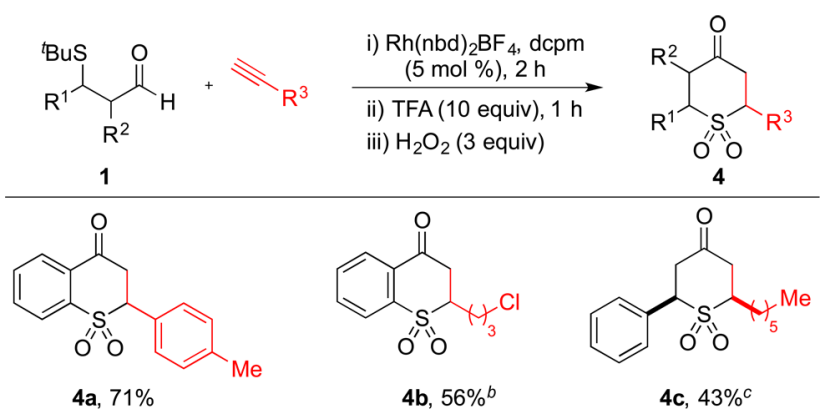

${ }^{a}$ Reaction conditions: (i) $\mathrm{Rh}(\mathrm{nbd}){ }_{2} \mathrm{BF}_{4}(5 \mathrm{~mol} \%)$, dcpm (5 mol \%), aldehyde 1a (1.0 equiv), alkyne (1.05 equiv), DCE $(1.0 \mathrm{M}), 55^{\circ} \mathrm{C}, 2$ h; (ii) TFA (10.0 equiv), $1 \mathrm{~h}, \mathrm{rt}$; (iii) $\mathrm{H}_{2} \mathrm{O}_{2}$ (3.0 equiv), rt. Isolated yields. ${ }^{b} \mathrm{H}_{2} \mathrm{O}_{2}$ (4.5 equiv). ${ }^{c}$ Crude dr 19:1.

approach we were able to access both thiochromen-4-ones and dihydrothiopyran-4-ones in good yields after three steps (5ad). As suggested by Chen, addition of excess NCS and pyridine provided the $\alpha$-chloro adducts $(6 \mathbf{a}-\mathbf{d})$. It is interesting to note the selective formation of dihydrothiopyranones $5 \mathbf{d}$ and $\mathbf{6 d}$ where oxidation has taken place adjacent to the aryl substituent.
Scheme 5. NCS-Mediated Formation of Thiochromenones and Dihydrothiopyranones ${ }^{a}$

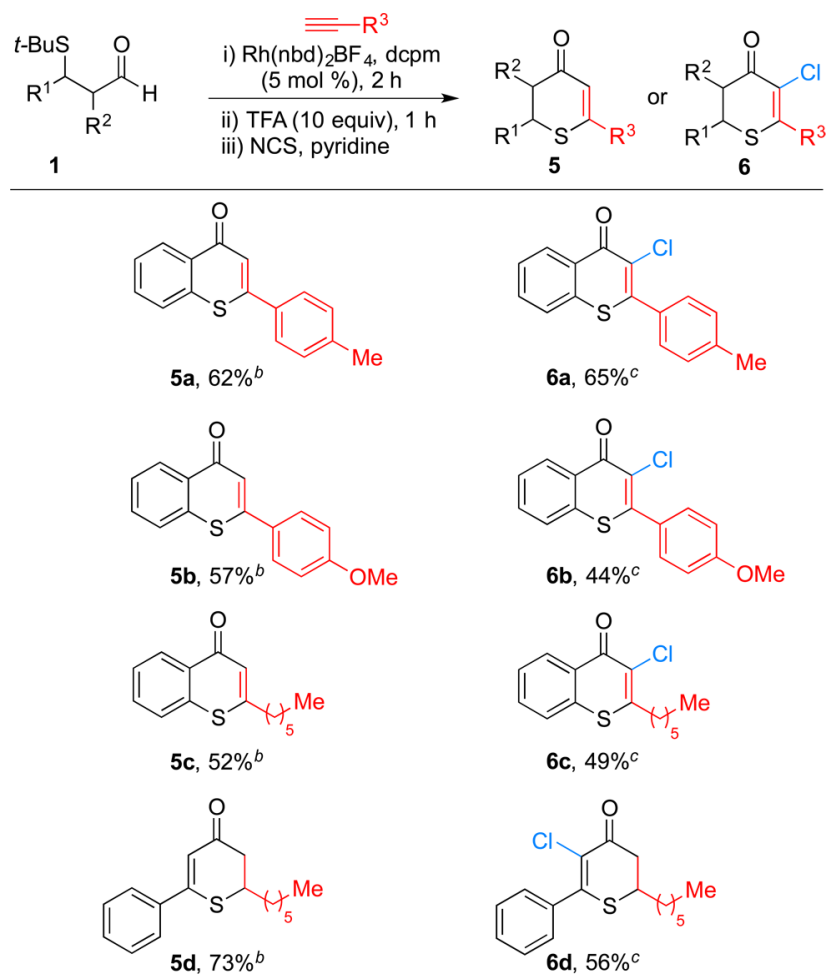

${ }^{a}$ Reaction conditions: (i) $\mathrm{Rh}(\mathrm{nbd}){ }_{2} \mathrm{BF}_{4}(5 \mathrm{~mol} \%)$, dcpm (5 mol \%), aldehyde (1.0 equiv), alkyne (1.05 equiv), DCE $(1.0 \mathrm{M}), 55{ }^{\circ} \mathrm{C}, 2 \mathrm{~h}$; (ii) TFA (10.0 equiv), $1 \mathrm{~h}, \mathrm{rt}^{b} \mathrm{NCS}$ ( 1.1 equiv), pyridine ( 1.1 equiv), DCE $(0.5 \mathrm{M}), \mathrm{rt}, 2 \mathrm{~h} .{ }^{c} \mathrm{NCS}$ (3.0 equiv), pyridine (3.0 equiv), DCE $(0.5 \mathrm{M}), \mathrm{rt}, 2 \mathrm{~h}$. Isolated yields.

In conclusion, we have developed a tandem alkyne hydroacylation/thio-conjugate addition sequence to synthesize thiochroman-4-one, hexahydro- $4 H$-thiochromen-4-one, and tetrahydrothiopyran-4-one derivatives, and their corresponding sulfones, from readily accessible starting materials. The addition of NCS and pyridine as a final step in the sequence allowed for the preparation of thiochromen-4-ones and dihydrothiopyran4-ones, as well as their chlorinated derivatives. The key hydroacylation step exploits $\beta$-( $t$-Bu-S)-substituted aldehydes as substrates for the first time.

\section{ASSOCIATED CONTENT}

\section{Supporting Information}

The Supporting Information is available free of charge on the ACS Publications website at DOI: 10.1021/acs.orglett.6b02909.

Experimental procedures and full characterization for all compounds (PDF)

\section{AUTHOR INFORMATION}

\section{Corresponding Author}

*E-mail: michael.willis@chem.ox.ac.uk.

Notes

The authors declare no competing financial interest. 


\section{ACKNOWLEDGMENTS}

The research leading to these results has received funding from the EPSRC and the People Programme (Marie Curie Actions) of the European Union's Seventh Framework Programme (FP7/2007-2013) under REA Grant Agreement No. 316955.

\section{REFERENCES}

(1) For reviews, see: (a) Jun, C.-H.; Jo, E.-A.; Park, J.-W. Eur. J. Org. Chem. 2007, 2007, 1869. (b) Willis, M. C. Chem. Rev. 2010, 110, 725. (c) Leung, J. C.; Krische, M. J. Chem. Sci. 2012, 3, 2202. (d) Ghosh, A.; Johnson, K. F.; Vickerman, K. L.; Walker, J. A.; Stanley, L. M. Org. Chem. Front. 2016, 3, 639.

(2) Non-chelating Rh examples: (a) Marder, T. B.; Roe, D. C.; Milstein, D. Organometallics 1988, 7, 1451. (b) Roy, A. H.; Lenges, C. P.; Brookhart, M. J. Am. Chem. Soc. 2007, 129, 2082.

(3) Non-chelating Ru examples: (a) Kondo, T.; Akazome, M.; Tsuji, Y.; Watanabe, Y. J. Org. Chem. 1990, 55, 1286. (b) Shibahara, F.; Bower, J. F.; Krische, M. J. J. Am. Chem. Soc. 2008, 130, 14120. (c) Omura, S.; Fukuyama, T.; Horiguchi, J.; Murakami, Y.; Ryu, I. J. Am. Chem. Soc. 2008, 130, 14094. (d) Williams, V. M.; Leung, J. C.; Patman, R. L.; Krische, M. J. Tetrahedron 2009, 65, 5024. (e) Chen, Q.-A.; Cruz, F. A.; Dong, V. M. J. Am. Chem. Soc. 2015, 137, 3157.

(f) Kim, J.; Yi, C. S. ACS Catal. 2016, 6, 3336.

(4) Non-chelating Co examples: (a) Lenges, C. P.; Brookhart, M. J. Am. Chem. Soc. 1997, 119, 3165. (b) Chen, Q. A.; Kim, D. K.; Dong, V. M. J. Am. Chem. Soc. 2014, 136, 3772.

(5) Non-chelating Ir examples: (a) Tsuda, T.; Kiyoi, T.; Saegusa, T. J. Org. Chem. 1990, 55, 2554. (b) Xiao, L.-J.; Fu, X.-N.; Zhou, M.-J.; Xie, J.-H.; Wang, L.-X.; Xu, X.-F.; Zhou, Q.-L. J. Am. Chem. Soc. 2016, 138, 2957.

(6) O-Chelation: (a) Kokubo, K.; Matsumasa, K.; Miura, M.; Nomura, M. J. Org. Chem. 1997, 62, 4564. (b) Imai, M.; Tanaka, M.; Tanaka, K.; Yamamoto, Y.; Imai-Ogata, N.; Shimowatari, M.; Nagumo, S.; Kawahara, N.; Suemune, H. J. Org. Chem. 2004, 69, 1144. (c) Coulter, M. M.; Kou, K. G. M.; Galligan, B.; Dong, V. M. J. Am. Chem. Soc. 2010, 132, 16330. (d) Parsons, S. R.; Hooper, J. F.; Willis, M. C. Org. Lett. 2011, 13, 998. (e) Murphy, S. K.; Petrone, D. A.; Coulter, M. M.; Dong, V. M. Org. Lett. 2011, 13, 6216.

(7) N-Chelation: (a) Jun, C. H.; Lee, D. Y.; Lee, H.; Hong, J. B. Angew. Chem., Int. Ed. 2000, 39, 3070. (b) Jun, C.-H.; Lee, H.; Hong, J.-B.; Kwon, B.-I. Angew. Chem., Int. Ed. 2002, 41, 2146. (c) Castaing, M.; Wason, S. L.; Estepa, B.; Hooper, J. F.; Willis, M. C. Angew. Chem., Int. Ed. 2013, 52, 13280. (d) Zhang, T.; Qi, Z.; Zhang, X.; Wu, L.; Li, X. Chem. - Eur. J. 2014, 20, 3283. (e) Fernández, M.; Castaing, M.; Willis, M. C. Chem. Sci. 2016, 7, doi: 10.1039/C6SC03066A.

(8) S-Chelation: (a) Willis, M. C.; McNally, S. J.; Beswick, P. J. Angew. Chem., Int. Ed. 2004, 43, 340. (b) Willis, M. C.; Woodward, R. L. J. Am. Chem. Soc. 2005, 127, 18012. (c) Moxham, G. L.; Randell-Sly, H. E.; Brayshaw, S. K.; Woodward, R. L.; Weller, A. S.; Willis, M. C. Angew. Chem., Int. Ed. 2006, 45, 7618. (d) Pawley, R. J.; Moxham, G. L.; Dallanegra, R.; Chaplin, A. B.; Brayshaw, S. K.; Weller, A. S.; Willis, M. C. Organometallics 2010, 29, 1717. (e) Prades, A.; Fernandez, M.; Pike, S. D.; Willis, M. C.; Weller, A. S. Angew. Chem., Int. Ed. 2015, 54, 8520. (f) Hooper, J. F.; Seo, S.; Truscott, F. R.; Neuhaus, J. D.; Willis, M. C. J. Am. Chem. Soc. 2016, 138, 1630.

(9) P-Chelation: Lee, H.; Jun, C. H. Bull. Korean Chem. Soc. 1995, 16, 66.

(10) C-Chelation: Vora, K. P.; Lochow, C. F.; Miller, R. G. J. Organomet. Chem. 1980, 192, 257.

(11) (a) Tanaka, K.; Shibata, Y.; Suda, T.; Hagiwara, Y.; Hirano, M. Org. Lett. 2007, 9, 1215. (b) Shibata, Y.; Tanaka, K. J. Am. Chem. Soc. 2009, 131, 12552. (c) Murphy, S. K.; Bruch, A.; Dong, V. M. Angew. Chem., Int. Ed. 2014, 53, 2455. (d) Murphy, S. K.; Bruch, A.; Dong, V. M. Chem. Sci. 2015, 6, 174.

(12) Regioselective examples: (a) Gonzalez-Rodriguez, C.; Pawley, R. J.; Chaplin, A. B.; Thompson, A. L.; Weller, A. S.; Willis, M. C. Angew. Chem., Int. Ed. 2011, 50, 5134. (b) Zhang, H.-J.; Bolm, C. Org. Lett.
2011, 13, 3900. (c) Poingdestre, S. J.; Goodacre, J. D.; Weller, A. S.; Willis, M. C. Chem. Commun. 2012, 48, 6354.

(13) Enantioselective examples: (a) Stemmler, R. T.; Bolm, C. Adv. Synth. Catal. 2007, 349, 1185. (b) Osborne, J. D.; Randell-Sly, H. E.; Currie, G. S.; Cowley, A. R.; Willis, M. C. J. Am. Chem. Soc. 2008, 130, 17232. (c) Gonzalez-Rodriguez, C.; Parsons, S. R.; Thompson, A. L.; Willis, M. C. Chem. - Eur. J. 2010, 16, 10950. (d) Phan, D. H. T.; Kou, K. G. M.; Dong, V. M. J. Am. Chem. Soc. 2010, 132, 16354.

(14) (a) Hooper, J. F.; Chaplin, A. B.; González-Rodríguez, C.; Thompson, A. L.; Weller, A. S.; Willis, M. C. J. Am. Chem. Soc. 2012, 134, 2906. (b) von Delius, M.; Le, C. M.; Dong, V. M. J. Am. Chem. Soc. 2012, 134, 15022. (c) Hooper, J. F.; Young, R. D.; Pernik, I.; Weller, A. S.; Willis, M. C. Chem. Sci. 2013, 4, 1568. (d) Hooper, J. F.; Young, R. D.; Weller, A. S.; Willis, M. C. Chem. - Eur. J. 2013, 19, 3125. (e) Arambasic, M.; Hooper, J. F.; Willis, M. C. Org. Lett. 2013, 15, 5162. (f) Niu, J.; Willis, M. C. Org. Chem. Front. 2016, 3, 625.

(15) Du, X.-W.; Stanley, L. M. Org. Lett. 2015, 17, 3276.

(16) (a) Clayden, J.; MacLellan, P. Beilstein J. Org. Chem. 2011, 7, 582. (b) Ramalingam, K.; Thyvelikakath, G. X.; Berlin, K. D.; Chesnut, R. W.; Brown, R. A.; Durham, N. N.; Ealick, S. E.; Van der Helm, D. J. Med. Chem. 1977, 20, 847. (c) Razdan, R. K.; Bruni, R. J.; Mehta, A. C.; Weinhardt, K. K.; Papanastassiou, Z. B. J. Med. Chem. 1978, 21, 643. (d) Nakazumi, H.; Ueyama, T.; Kitao; Teijiro. J. Heterocycl. Chem. 1984, 21, 193. (e) Al Nakib, T.; Bezjak, V.; Meegan, M. J.; Chandy, R. Eur. J. Med. Chem. 1990, 25, 455. (f) Lee, J. I.; Lee, J.-H. Food Sci. Biotechnol. 2014, 23, 957. (g) Sangeetha, S.; Muthupandi, P.; Sekar, G. Org. Lett. 2015, 17, 6006. (h) Shen, C.; Spannenberg, A.; Wu, X.-F. Angew. Chem., Int. Ed. 2016, 55, 5067.

(17) Lee, J. I. Bull. Korean Chem. Soc. 2008, 29, 1263.

(18) Majhail, M. K.; Ylioja, P. M.; Willis, M. C. Chem. - Eur. J. 2016, 22, 7879 .

(19) Kobayashi, K.; Kobayashi, A.; Ezaki, K. Heterocycles 2012, 85, 1997.

(20) (a) Chaplin, A. B.; Hooper, J. F.; Weller, A. S.; Willis, M. C. J. Am. Chem. Soc. 2012, 134, 4885. (b) Pernik, I.; Hooper, J. F.; Chaplin, A. B.; Weller, A. S.; Willis, M. C. ACS Catal. 2012, 2, 2779.

(21) Chen, C. H.; Reynolds, G. A.; Van Allan, J. A. J. Org. Chem. 1977, 42, 2777. 